\title{
Diversidade de peixes do reservatório da UHE Escola Engenharia Mackenzie (Capivara), Rio Paranapanema, bacia do alto rio Paraná, Brasil, e a importância dos grandes tributários na sua manutenção
}

\author{
Ana Cecília Hoffmann ${ }^{1}$, Mario L. Orsi ${ }^{2} \&$ Oscar A. Shibatta ${ }^{1}$ \\ 1. Pós-Graduação em Ciências Biológicas, Centro de Ciências Biológicas, Universidade Estadual de Londrina, Rod. Celso Garcia Cid, \\ 86.051-990 Londrina, PR, Brasil. (cecishoffmann@ hotmail.com) \\ 2. Departamento de Biologia Animal e Vegetal, Centro de Ciências Biológicas, Universidade Estadual de Londrina, Rod. Celso Garcia \\ Cid, 86051-990 Londrina, PR, Brasil. (orsi@uel.br)
}

\begin{abstract}
Fish diversity in the UHE Escola Engenharia Mackenzie (Capivara) reservoir, Paranapanema River, upper Rio Paraná basin, Brazil, and the importance of large tributaries in its maitenance. Fish samples were taken in four stretches along a gradient formed by the UHE Escola Engenharia Mackenzie (Capivara) reservoir. The diversity of species was analyzed by the constance, index of Shannon-Wiener and similarity of the composition among stretches through the Jaccard's coefficient. The reservoir presents a richness of fishes composed by 67 species distributed in 5 orders; $47 \%$ of the species are constant, $15 \%$ are accessory and 28\% are accidental. The highest values of Shannon-Wiener diversity and Jaccard's similarity were obtained in Cinzas and Tibagi, wich are the most distant points from the dam and which present large tributaries. The lowest values were found in Cruzália and Porecatu, which respectively correspond to the stretches with lotic and semi-lotic environmental characteristics. This work demonstrates the extreme importance of tributaries for the maintenance of species diversity in a reservoir, as a result of the preservation of the original characteristics of lotic systems in those river stretches and consequent reduction of the damming impact.
\end{abstract}

KEYWORDS. Fish assemblages, dams, freshwater fish, Neotropical, reservoir.

RESUMO. As espécies foram coletadas em quatro trechos ao longo de um gradiente formado pelo reservatório UHE Escola Engenharia Mackenzie (Capivara). A diversidade de espécies foi analisada pela constância, índice de Shannon-Wiener e similaridade da composição entre trechos através do coeficiente de Jaccard. O reservatório tem uma riqueza de peixes composta por 67 espécies incluídas em 5 ordens; $47 \%$ das espécies são constantes, $15 \%$ são acessórias e $28 \%$ são acidentais. Os maiores índices de diversidade de Shannon-Wiener e similaridade de Jaccard foram obtidos em Cinzas e Tibagi, que são os trechos mais distantes da barragem e que apresentam grandes tributários. Os menores índices foram encontrados em Cruzália e Porecatu, os quais correspondem aos trechos com características, respectivamente, de ambientes lótico e semi-lótico. Este trabalho demonstra a extrema importância da presença dos tributários para a manutenção da diversidade das espécies em um reservatório, em função da preservação das características originais do sistema lótico naqueles trechos e conseqüente redução do impacto do represamento.

PALAVRAS-CHAVE. Assembléia de peixes, barragens, peixes de água doce, Neotropical, reservatório.

O aproveitamento hidrelétrico do rio Paranapanema iniciou-se em 1951, com as obras da Usina Hidrelétrica (UHE) Lucas Nogueira Garcez em Salto Grande, SP (CESP, 1993), até atingir o número atual de dez usinas em operação. Estas formam uma sequiência de ambientes impactados onde, segundo Agostinho \& Gomes (1997), ocorrem mudanças drásticas no regime hídrico, alterações na composição e diminuição da riqueza de espécies autóctones.

Considerando que a Região Neotropical possui a mais rica ictiofauna do mundo, com estimativa de cerca de 8.000 espécies (SCHAEFFER, 1998), e que essas ocorrem preferencialmente em ambientes lóticos, esta riqueza está sendo ameaçada pela construção de numerosos reservatórios (FERREIRA, 1993; SANTOS, 1995). Portanto, cada vez mais há necessidade de diagnósticos corretos dos fatores que influenciam as comunidades ícticas e de estudos aprofundados de como elas se comportam, para que medidas de conservação possam ser tomadas.

Recentemente, foi observado que a diversidade de Gymnotiformes do rio Amazonas aumenta abaixo e na foz dos principais tributários (COX-FERNANDEs et al., 2004). $\mathrm{O}$ mesmo deve ocorrer para a assembléia de peixes no médio Paranapanema, e a identificação desse fenômeno é importante, uma vez que serviria como informação relevante ao planejamento de futuras hidrelétricas, em que seria exigida a presença de grandes afluentes para auxiliar na manutenção da diversidade de peixes da região.

Portanto, este trabalho teve por objetivo analisar a diversidade da ictiofauna em diferentes trechos ao longo do reservatório da UHE Escola Engenharia Mackenzie (Capivara), no rio Paranapanema, com diferentes graus de represamento e influência dos principais tributários.

\section{MATERIAL E MÉTODOS}

O rio Paranapanema, afluente da margem esquerda do rio Paraná, participa do sistema hidrográfico conhecido como bacia do Alto Paraná. Percorre uma extensão de aproximadamente $660 \mathrm{~km}$ (SAMPAIO, 1944), estabelecendo a divisa natural entre os estados de São Paulo e Paraná ao longo de 329,9 km (MAACK, 1981). No Paraná os tributários de maior porte são os rios Itararé, Cinzas, Tibagi e Pirapó, enquanto que em São Paulo há um único grande tributário que é o rio Pardo.

O reservatório de Capivara foi formado em 1977, com a construção da UHE Escola Engenharia Mackenzie, e localiza-se no curso médio do rio Paranapanema. Este 
reservatório possui $100 \mathrm{~km}$ de comprimento e larguras que variam de 800 a 10.000 metros. A profundidade varia de $70 \mathrm{~m}$ próximo à barragem até $6 \mathrm{~m}$ à montante, próximo à barragem da UHE Canoas I. Dois dos principais tributários do rio Paranapanema, Tibagi e Cinzas, deságuam nesse reservatório.

Neste trabalho foi adotado o termo trecho de coleta, ou de amostragem, para uma extensão de aproximadamente
$1.000 \mathrm{~m}$ onde os aparelhos de coleta foram instalados. Os trechos de amostragem foram selecionados ao longo de um gradiente de distância formado entre a barragem e o trecho mais distante de sua influência, e pela presença ou ausência de tributários (Fig. 1). Em cada trecho de coleta foram tomados dados de transparência, com o disco de Secchi, e de profundidade. Essas e outras características físicas estão apresentadas na tabela I.

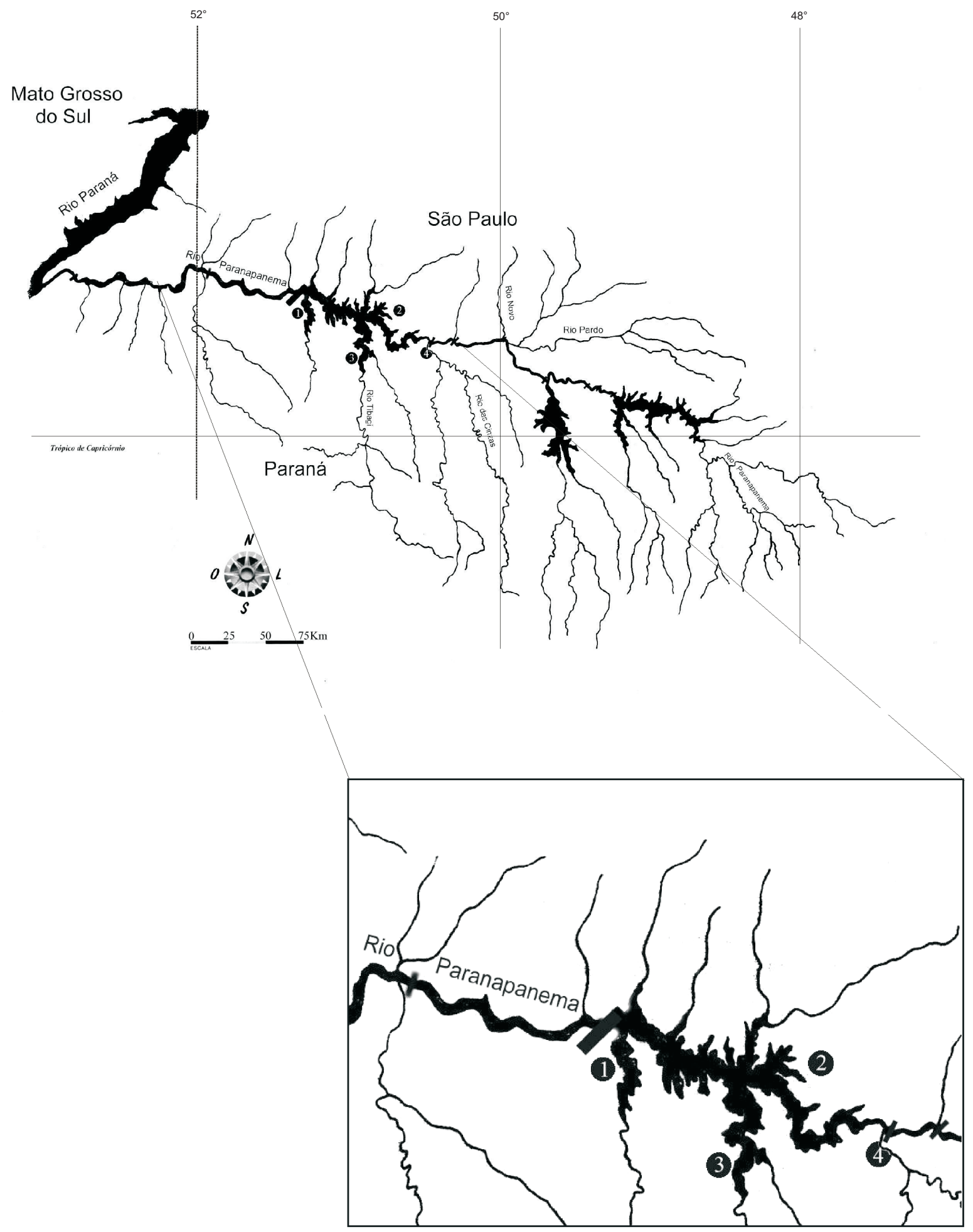

Fig. 1. Trechos de amostragem de peixes da UHE Escola Engenharia Machenzie (Capivara), rio Paranapanema, Brasil (maio de 2001 a abril de 2002). 1, Porecatu; 2, Cruzália; 3, Tibagi; 4, Cinzas. Modificado de Zocchi (2002). 
Tabela I. Características físicas e localização geográfica das estações de amostragem no reservatório de UHE Escola Engenharia Mackenzie (Capivara), rio Paranapanema, Brasil (maio de 2001 a abril de 2002).

\begin{tabular}{lccccc}
\hline Estação & \multicolumn{1}{c}{ Coordenadas } & Veloc. corrente & Profundidade & Transparência & Vegetação ciliar \\
\hline Porecatu & $\left(22^{\circ} 40^{\prime} 44^{\prime \prime} \mathrm{S} ; 51^{\circ} 19^{\prime} 55^{\prime \prime} \mathrm{W}\right)$ & Semi-lóticas & Entre 15 e $70 \mathrm{~m}$ & Aprox. $2 \mathrm{~m}$ & Escassa \\
Cruzália & $\left(22^{\circ} 46^{\prime} 14^{\prime \prime} \mathrm{S} ; 50^{\circ} 50^{\prime} 34^{\prime \prime} \mathrm{W}\right)$ & Lênticas & Média de $6 \mathrm{~m}$ & Aprox. $1 \mathrm{~m}$ & Ausente \\
Tibagi & $\left(23^{\circ} 01^{\prime} 16^{\prime \prime} \mathrm{S} ; 50^{\circ} 57^{\prime} 13^{\prime \prime} \mathrm{W}\right)$ & Semi-lóticas & Até $25 \mathrm{~m}$ & 1,0 a $0,7 \mathrm{~m}$ & Escassa \\
Cinzas & $\left(22^{\circ} 56^{\prime} 16^{\prime \prime} \mathrm{S} ; 50^{\circ} 31^{\prime} 37^{\prime \prime} \mathrm{W}\right)$ & Lótica & 0,8 a $5,2 \mathrm{~m}$ & 0,6 a $9,2 \mathrm{~m}$ & Pouco expressiva \\
\hline
\end{tabular}

Em cada uma das localidades selecionadas, foram feitas quatro coletas, entre maio de 2001 e abril de 2002, com um intervalo de três meses. Para a captura dos peixes foram utilizadas trinta e uma redes de espera de malhas entre 1,3 e 10 centímetros entre nós opostos, totalizando $1.527 \mathrm{~m}^{2}$ de área. As redes foram armadas tanto na região marginal como na calha do rio, de forma paralela e perpendicular à margem. Cada amostragem teve duração máxima de 24 horas, divididas em duas revisões nos períodos do amanhecer e do anoitecer. Duas pessoas também utilizaram redes de arrasto, tarrafas e peneiras, com padronização de duas horas de esforço por coleta para cada aparelho. Dois covos de $45 \mathrm{~cm}$ de diâmetro e $120 \mathrm{~cm}$ de comprimento permaneceram 24 horas na região marginal do rio. Os peixes foram fixados com formol a $10 \%$ e posteriormente transferidos para álcool $70 \%$, identificados e depositados como material testemunho no Museu de Zoologia da Universidade Estadual de Londrina.

Foram atribuídos valores de constância para cada espécie, calculados a partir da fórmula $\mathrm{C}=\mathrm{p} \times 100 / \mathrm{P}$ (DAJOZ, 1978) onde C é o valor de constância da espécie, p é o número de trechos que contêm a espécie e $\mathrm{P}$ é o número total de trechos. As espécies foram consideradas constantes quando apresentaram $\mathrm{C}>50$, acessórias quando $25 \geq \mathrm{C} \leq 50$ e acidentais quando $\mathrm{C}<25$.

Foram calculados os índices de diversidade de Shannon-Wiener (H'), equitabilidade e dominância para cada localidade, utilizando-se a abundância média das espécies coletadas tanto pelos aparelhos passivos quanto ativos, pois a padronização dos procedimentos de coleta em todos os trechos permite realizar comparações sem a necessidade de separar os esforços de captura. As variâncias dos índices de diversidade foram comparadas com o teste $t$ de Student (MaGurRan, 1988) para demonstrar diferenças entre os trechos. A análise de similaridade entre os trechos foi apresentada em forma de dendrograma utilizando-se UPGMA (associação por médias aritméticas não-ponderadas), conforme SNEATH \& SOKAL (1973) sobre o índice de BrayCurtis aplicado simultaneamente aos dados de diversidade, equitabilidade e dominância. Essas análises foram feitas com o programa Past (HAMmer et al., 2003).

\section{RESULTADOS}

Foram coletadas 67 espécies de cinco ordens e 20 famílias distribuídas da seguinte maneira: 32 espécies de Characiformes, 22 de Siluriformes, 7 de Perciformes, 5 de Gymnotiformes e uma de Synbranchiformes (Tab. II). O trecho com maior riqueza de espécies foi Cinzas (62 espécies), seguido por Tibagi
(43), Porecatu (31) e Cruzália (24).

Oito espécies (Acestrorhynchus lacustris, Astyanax altiparanae, Apareiodon affinis, Moenkhausia intermedia, Steindachnerina insculpta, Loricariichthys platymetopon, Plagioscion squamosissimus e Cichla monoculus) representaram $59 \%$ do total de indivíduos coletados. Entre estas, Astyanax altiparanae foi a que apresentou maior número de indivíduos e esteve presente nos quatro trechos. Cinco espécies (Cichla monoculus, Loricariichthys platymetopon, Plagioscion squamosissimus, Sorubim lima e Tilapia rendalli) são exóticas.

Para as demais espécies o resultado de constância apontou que 19 estiveram presentes em todos os trechos amostrados e 13 em pelo menos três dos quatro trechos (Tab. II) totalizando $47 \%$ de espécies constantes. Para os dados de constância têmse ainda que $15 \%$ das espécies são acessórias e $38 \%$ são acidentais, ou seja, presentes em um trecho somente.

$\mathrm{O}$ trecho Cinzas foi o que apresentou o maior número de espécies exclusivas, tais como Apteronotus albifrons, Astyanax eigenmanniorum, Piabina argentea, Bryconamericus stramineus, Serrapinnus stenodon, Cyphocharax nagelii, Hypostomus albopunctatus, $H$. iheringii, $H$. margaritifer, $H$. nigromaculatus, Hypostomus sp. I, Hypostomus sp. III, Hypostomus sp. IV, Loricaria prolixa, Pimelodella avanhandavae, Salminus brasiliensis, Schizodon altoparanae, Sorubim lima e Synbranchus marmoratus.

Os maiores índices de diversidade de ShannonWiener $\left(\mathrm{H}^{\prime}\right)$ foram obtidos em Cinzas, seguido por Tibagi, ao passo que o menor índice foi obtido em Cruzália (Tab. III). Analisando-se o H'máx de cada trecho, é possível notar que Cinzas apresentou o maior índice de diversidade, enquanto que Cruzália o menor. $\mathrm{O}$ maior valor de equitabilidade foi observado em Tibagi, seguido por Cinzas, Cruzália e Porecatu. Apesar da variação desse índice ter sido pequena entre os trechos (de 0,75 a 0,80 ), quando se analisa a dominância de espécies nota-se os valores são representativos e que a relação é inversa $\left(\mathrm{r}^{2}=0,80\right)$, com menor valor de dominância no trecho Cinzas, seguido por Tibagi, Porecatu e Cruzália. Os índices de diversidade foram significativamente diferentes entre os trechos (teste $t, \mathrm{p}<0,001$ ), exceto entre Porecatu e Cruzália, que apresentaram os menores índices, e entre Tibagi e Cinzas, que tiveram os maiores índices (Tab. IV).

No dendrograma pode-se observar dois grupos 
Tabela II. Relação taxonômica, números total e médio (entre parênteses) de indivíduos e constância das espécies capturadas no reservatório da UHE Escola Engenharia Mackenzie (Capivara), rio Paranapanema, Brasil (maio de 2001 a abril de 2002) (Ace, acessória; Aci, acidental; C, constante; Cin, Cinzas; Cru, Cruzália; Por, Porecatu; Tib, Tibagi).

\begin{tabular}{|c|c|c|c|c|c|c|}
\hline Táxons & Por & Cru & Tib & Cin & Total & Constânci \\
\hline $\begin{array}{l}\text { CHARACIFORMES } \\
\text { Erythrinidae }\end{array}$ & & & & & & \\
\hline $\begin{array}{l}\text { Hoplias malabaricus (Bloch, 1794) } \\
\text { Acestrorhynchidae }\end{array}$ & $3(0,75)$ & $9(2,25)$ & $29(7,25)$ & $17(4,25)$ & 58 & $\mathrm{C}$ \\
\hline $\begin{array}{l}\text { Acestrorhynchus lacustris (Lütken, 1875) } \\
\text { Characidae }\end{array}$ & $36(9)$ & $81(20,25)$ & $43(10,75)$ & $69(17,25)$ & 229 & $\mathrm{C}$ \\
\hline Aphyocharax anisitsi (Eigenmann \& Kennedy, 1903) & 0 & $2(0,5)$ & $2(0,5)$ & $86(21,5)$ & 90 & $\mathrm{C}$ \\
\hline Serrapinnus notomelas (Eigenmann, 1915) & 0 & $6(1,5)$ & $9(2,25)$ & $23(5,75)$ & 38 & $\mathrm{C}$ \\
\hline Serrapinnus stenodon (Eigenmann, 1915) & 0 & 0 & 0 & $1(0,25)$ & 1 & Aci \\
\hline Galeocharax knerii (Steindachner, 1879) & $2(0,5)$ & $1(0,25)$ & $5(1,25)$ & $7(1,75)$ & 15 & $\mathrm{C}$ \\
\hline Astyanax altiparanae Garutti \& Britski, 2000 & $242(60,5)$ & $107(26,75)$ & $22(5,5)$ & $306(76,5)$ & 677 & $\mathrm{C}$ \\
\hline Astyanax eigenmanniorum (Cope, 1894) & 0 & 0 & 0 & $1(0,25)$ & 1 & Aci \\
\hline Piabina argentea Reinhardt, 1867 & 0 & 0 & 0 & $14(3,5)$ & 14 & Aci \\
\hline Bryconamericus stramineus Eigenmann, 1908 & 0 & 0 & 0 & $30(7,5)$ & 30 & Aci \\
\hline Hemigrammus marginatus Ellis, 1911 & $6(1,5)$ & 0 & $22(5,5)$ & $95(23,75)$ & 123 & $\mathrm{C}$ \\
\hline Hyphessobrycon eques (Steindachner, 1882) & $9(2,25)$ & 0 & $54(13,5)$ & $72(18)$ & 135 & $\mathrm{C}$ \\
\hline Moenkhausia intermedia Eigenmann, 1908 & $34(8,5)$ & $57(14,25)$ & $72(18)$ & $16(4)$ & 179 & $\mathrm{C}$ \\
\hline Metynnis maculatus (Kner, 1860) & 0 & $87(21,75)$ & $63(15,75)$ & $1(0,25)$ & 151 & $\mathrm{C}$ \\
\hline Serrasalmus maculatus Kner, 1858 & $10(2,5)$ & $17(4,25)$ & $33(8,25)$ & $41(10,25)$ & 101 & $\mathrm{C}$ \\
\hline Salminus brasiliensis Cuvier, 1850 & 0 & 0 & 0 & $6(1,5)$ & 6 & Aci \\
\hline $\begin{array}{l}\text { Triportheus angulatus Spix \& Agassiz, } 1829 \\
\text { Crenuchidae }\end{array}$ & 0 & $12(3)$ & $57(14,25)$ & $2(0,5)$ & 71 & $\mathrm{C}$ \\
\hline $\begin{array}{l}\text { Characidium zebra Eigenmann, } 1909 \\
\text { Anostomidae }\end{array}$ & 0 & 0 & $2(0,5)$ & $24(6)$ & 26 & Ace \\
\hline Leporellus vittatus (Kner, 1859) & $6(1,5)$ & 0 & $1(0,25)$ & $1(0,25)$ & 8 & $\mathrm{C}$ \\
\hline Leporinus elongatus Valenciennes, 1850 & $1(0,25)$ & $1(0,25)$ & $8(2)$ & $9(2,25)$ & 19 & $\mathrm{C}$ \\
\hline Leporinus friderici (Bloch, 1794) & $4(0,25)$ & 0 & $11(2,75)$ & $3(0,75)$ & 18 & $\mathrm{C}$ \\
\hline Leporinus obtusidens (Valenciennes, 1836) & $1(0,25)$ & $1(0,25)$ & $6(1,5)$ & $1(0,25)$ & 9 & $\mathrm{C}$ \\
\hline Leporinus striatus Kner, 1858 & 0 & 0 & 0 & $9(2,25)$ & 9 & Aci \\
\hline Schizodon altoparanae Garavello \& Britski, 1990 & 0 & 0 & 0 & $4(1)$ & 4 & Aci \\
\hline Schizodon intermedius Garavello \& Britski, 1990 & 0 & $1(0,25)$ & $22(5,5)$ & $25(6,25)$ & 48 & $\mathrm{C}$ \\
\hline $\begin{array}{l}\text { Schizodon nasutus } \quad \text { Kner, } 1858 \\
\text { Parodontidae }\end{array}$ & $45(11,25)$ & $10(2,5)$ & $12(3)$ & $30(7,5)$ & 97 & $\mathrm{C}$ \\
\hline Apareiodon affinis (Steindachner, 1879) & $130(32,5)$ & $5(1,25)$ & $1(0,25)$ & $76(19)$ & 212 & $\mathrm{C}$ \\
\hline $\begin{array}{l}\text { Apareiodon piracicabae (Eigenmann, 1907) } \\
\text { Prochilodontidae }\end{array}$ & $16(4)$ & $26(6,5)$ & $1(0,25)$ & $88(22)$ & 131 & $\mathrm{C}$ \\
\hline $\begin{array}{l}\text { Prochilodus lineatus (Valenciennes, 1836) } \\
\text { Curimatidae }\end{array}$ & 0 & 0 & $2(0,5)$ & $8(2)$ & 10 & Ace \\
\hline Cyphocharax modestus (Fernández-Yépez, 1948) & $1(0,25)$ & 0 & $2(0,5)$ & $4(1)$ & 7 & $\mathrm{C}$ \\
\hline Cyphocharax nagelii (Steindachner, 1881) & 0 & 0 & 0 & $1(0,25)$ & 1 & Aci \\
\hline $\begin{array}{l}\text { Steindachnerina insculpta (Fernández-Yépez, 1948) } \\
\text { SILURIFORMES }\end{array}$ & $48(12)$ & $113(28,25)$ & $40(10)$ & $136(34)$ & 337 & $\mathrm{C}$ \\
\hline $\begin{array}{l}\text { Callichthyidae } \\
\text { Hoplosternum littorale (Hancock, 1828) } \\
\text { Loricariidae }\end{array}$ & $31(7,75)$ & 0 & 0 & 0 & 31 & Aci \\
\hline Hypostomus albopunctatus (Regan, 1908) & 0 & 0 & 0 & $1(0,25)$ & 1 & Aci \\
\hline Hypostomus ancistroides (Ihering, 1911) & 0 & 0 & $21(5,25)$ & $49(12,25)$ & 70 & Ace \\
\hline Hypostomus iheringii (Regan, 1908) & 0 & 0 & 0 & $1(0,25)$ & 1 & Aci \\
\hline Hypostomus margaritifer (Regan, 1908) & 0 & 0 & 0 & $1(0,25)$ & 1 & Aci \\
\hline Hypostomus nigromaculatus (Gosline, 1947) & 0 & 0 & 0 & $1(0,25)$ & 1 & Aci \\
\hline Hypostomus strigaticeps (Regan, 1908) & $1(0,25)$ & 0 & 0 & 0 & 1 & Aci \\
\hline Loricaria prolixa Isbrücker \& Nijssen, 1978 & 0 & 0 & 0 & $2(0,5)$ & 2 & Aci \\
\hline $\begin{array}{l}\text { Loricariichthys platymetopon Isbrücker \& Nijssen, } 1979 \\
\text { Pimelodidae }\end{array}$ & $18(4,5)$ & $228(57)$ & $148(37)$ & $95(23,75)$ & 489 & $\mathrm{C}$ \\
\hline Iheringichthys labrosus (Lütken, 1874) & $24(6)$ & $23(5,75)$ & $20(5)$ & $17(4,25)$ & 84 & $\mathrm{C}$ \\
\hline Pimelodus maculatus Lacépède, 1803 & $13(3,25)$ & $70(17,5)$ & $28(7)$ & $20(5)$ & 131 & $\mathrm{C}$ \\
\hline Pinirampus pirinampu (Spix \& Agassix, 1829) & $8(2)$ & 0 & $6(1,5)$ & $6(1,5)$ & 20 & $\mathrm{C}$ \\
\hline $\begin{array}{l}\text { Sorubim lima (Bloch \& Schneider, 1801) } \\
\text { Heptapteridae }\end{array}$ & 0 & 0 & 0 & $2(0,5)$ & 2 & Aci \\
\hline $\begin{array}{l}\text { Pimelodella avanhandavae Eigenmann, } 1917 \\
\text { Doradidae }\end{array}$ & 0 & 0 & 0 & $6(1,5)$ & 6 & Aci \\
\hline $\begin{array}{l}\text { Rhinodoras dorbignyi (Kroyer, 1855) } \\
\text { Ageneiosidae }\end{array}$ & 0 & 0 & $3(0,75)$ & $13(3,25)$ & 16 & Ace \\
\hline $\begin{array}{l}\text { Ageneiosus valenciennesi Bleeker, } 1864 \\
\text { Auchenipteridae }\end{array}$ & 0 & 0 & $1(0,25)$ & $7(1,75)$ & 8 & Ace \\
\hline $\begin{array}{l}\text { Tatia neivai (Ihering, 1930) } \\
\text { GYMNOTIFORMES }\end{array}$ & 0 & 0 & $11(2,75)$ & $6(1,5)$ & 17 & Ace \\
\hline Gymnotidae & & & & & & \\
\hline Gymnotus carapo Linnaeus, 1758 & $1(0,25)$ & 0 & $6(1,5)$ & $11(2,75)$ & 18 & $\mathrm{C}$ \\
\hline
\end{tabular}


Tabela II. (continuação)

Apteronotidae

Apteronotus albifrons (Linnaeus, 1766)

Porotergus ellisi Arámburu, 1957

Sternopygidae

Sternopygus macrurus (Bloch \& Schneider, 1801)

Eigenmannia virescens (Valenciennes, 1842)

PERCIFORMES

Cichlidae

Cichla monoculus (Spix \& Agassiz, 1831)

Cichlasoma paranaense Kullander, 1983

Crenicichla britskii Kullander, 1982

Crenicichla niederleinii (Holmberg, 1891)

Geophagus brasiliensis Kner, 1865

Tilapia rendalli (Boulenger, 1897)

Sciaenidae

Plagioscion squamosissimus (Heckel, 1840)

SYNBRANCHIFORMES

Synbranchidae

Synbranchus marmoratus Bloch, 1795

Total Geral

\begin{tabular}{|c|c|c|c|c|c|}
\hline 0 & 0 & 0 & $1(0,25)$ & 1 & Aci \\
\hline 0 & 0 & $1(0,25)$ & $18(4,5)$ & 19 & Ace \\
\hline 0 & 0 & $5(1,25)$ & $1(0,25)$ & 6 & Ace \\
\hline $3(0,75)$ & 0 & $6(1,5)$ & $14(3,5)$ & 23 & $\mathrm{C}$ \\
\hline $179(44,75)$ & $9(2,25)$ & $3(0,75)$ & $2(0,5)$ & 193 & $\mathrm{C}$ \\
\hline 0 & 0 & $10(2,5)$ & 0 & 10 & Aci \\
\hline $14(3,5)$ & $1(0,25)$ & $7(1,75)$ & $5(1,25)$ & 27 & $\mathrm{C}$ \\
\hline $18(4,5)$ & $6(1,5)$ & $7(1,75)$ & $29(7,25)$ & 60 & $\mathrm{C}$ \\
\hline $22(5,5)$ & 0 & 0 & $1(0,25)$ & 23 & Ace \\
\hline $7(1,75)$ & 0 & 0 & 0 & 7 & Aci \\
\hline $69(17,25)$ & $105(26,25)$ & $168(42)$ & $53(13,25)$ & 395 & $\mathrm{C}$ \\
\hline 0 & 0 & 0 & $18(4,5)$ & $\begin{array}{c}18 \\
4482\end{array}$ & Aci \\
\hline
\end{tabular}

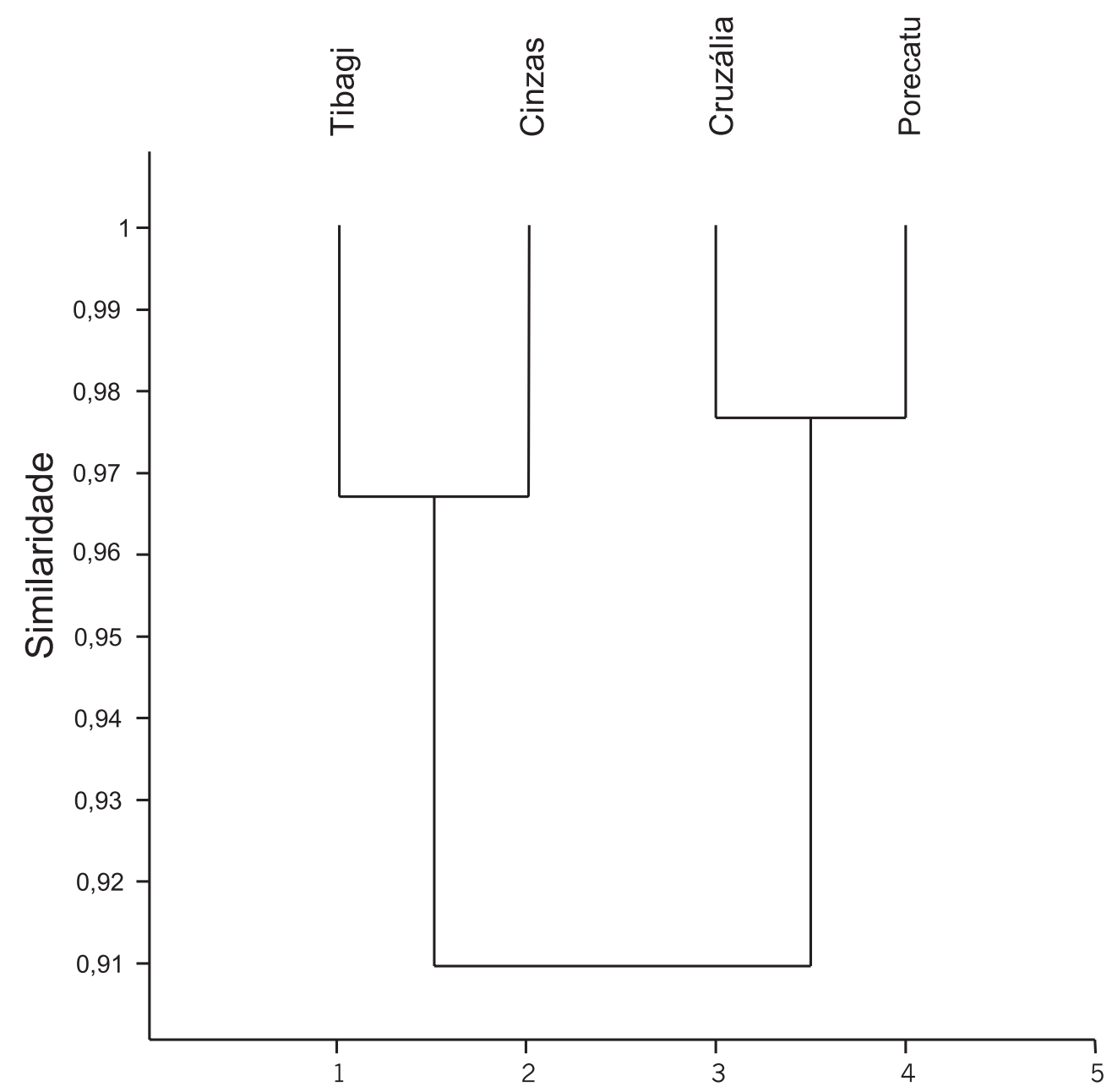

Fig. 2. Dendrograma de similaridade de Bray-Curtis, elaborado a partir dos dados de diversidade (H), equitabilidade e dominância das amostras de peixes em quatro trechos da represa Capivara, rio Paranapanema, Brasil (maio de 2001 a abril de 2002 ).

distintos formados através da alta similaridade de espécies de peixes entre os trechos Cinzas e Tibagi, seguido da similaridade entre Porecatu e Cruzália (Fig. 2). Estes dois grupos são formados pela semelhança no grande número de espécies em Cinzas e Tibagi, nove das quais exclusivas destes dois trechos
(Characidium zebra, Prochilodus lineatus, Hypostomus ancistroides, Hypostomus sp. VII, Rhinodoras dorbignyi, Ageneiosus valenciennesi, Tatia neivai, Porotergus ellisi e Sternopygus macrurus), e pelo número menor de espécies com grande abundância em Porecatu e Cruzália, este último 
Tabela III. Riqueza de espécies de peixe, índice de diversidade máxima (H'máx) e índices de diversidade de Shannon-Wiener (H') obtidos para cada trecho de amostragem com suas respectivas eqüitabilidades (E) e variância (Var H') na UHE Escola Engenharia Mackenzie (Capivara), rio Paranapanema, Brasil (maio de 2001 a abril de 2002).

\begin{tabular}{cccccc}
\hline & Riqueza & H'máx & H' & E & Var H' \\
\hline Porecatu & 31 & 3,43 & 2,58 & 0,75 & 0,0051986 \\
Cruzália & 24 & 3,18 & 2,44 & 0,77 & 0,0033889 \\
Tibagi & 43 & 3,76 & 2,98 & 0,79 & 0,0050984 \\
Cinzas & 62 & 4,13 & 3,19 & 0,78 & 0,0033299 \\
\hline
\end{tabular}

Tabela IV. Valores de $t$ e P da comparação das variâncias dos índices de diversidade de Shannon-Wiener entre dois trechos de coleta na UHE Escola Engenharia Mackenzie (Capivara), rio Paranapanema, Brasil (maio de 2001 a abril de 2002). (*, indica diferenças significativas)

\begin{tabular}{lcc}
\hline Trechos de amostragem & $\mathrm{t}$ & $\mathrm{P}$ \\
\hline Porecatu x Cinzas* & $-6,8081$ & $2,69 \times 10^{-11}$ \\
Porecatu x Tibagi* & $-3,6364$ & $3,06 \times 10^{-4}$ \\
Porecatu x Cruzália & 1,3073 & $1,91 \times 10^{-1}$ \\
Cinzas x Tibagi & $-2,7334$ & $6,48 \times 10^{-3}$ \\
Cinzas x Cruzália* & 9,1484 & $9,04 \times 10^{-14}$ \\
Tibagi x Cruzália* & $-5,4158$ & $9,80 \times 10^{-8}$ \\
\hline
\end{tabular}

com nenhuma espécie exclusiva e uma riqueza quase toda formada por espécies que estão presentes em todos os trechos.

\section{DISCUSSÃO}

Com 60 espécies identificadas neste trabalho, houve expressiva ampliação do número de espécies conhecidas para o rio Paranapanema, pois nos últimos levantamentos realizados por CARVALHO et al. (1998) e Dias \& GaRAVELlo (1998), haviam sido registradas apenas 32 e 51 espécies respectivamente. Analisando as listas de espécies apresentadas nesses trabalhos, verifica-se que 33 ainda não haviam sido coletadas no rio.

A dominância de Characiformes e Siluriformes observada em Capivara, nos quatro trechos de coleta, concorda com o descrito para os ambientes neotropicais (Lowe-McConNell, 1999). A destacada participação da família Characidae, entre os Characiformes, é decorrente da ampla distribuição de suas espécies em água doce, além desta família incluir a maioria das espécies de peixes de águas interiores do Brasil (BRITSKI, 1972). Além disso, entre os Characiformes há um grande predomínio de espécies de pequeno porte e/ou capazes de concluir seu ciclo de vida em ambientes lênticos, como evidenciada por outros trabalhos (Agostinho et al., 1992; ARAúJo \& SANTOS, 2001; Orsi et al., 2002). Espécies como Astyanax altiparanae apresentam grande flexibilidade de hábitos alimentares e capacidade de reprodução em diversos habitats (Bennemann et al., 2000), o que deve ter permitido sua expressiva distribuição e abundância no reservatório de Capivara. Segundo Agostinho et al. (1999), a redução da média do tamanho corporal das espécies de peixes que constituem a assembléia íctica de um reservatório é um dos mais notáveis impactos destes empreendimentos. Isto pode ocorrer por oportunismo destas espécies frente às mudanças na composição dos recursos disponíveis (AgostinHo, 1992).

O trecho Cinzas apresentou o maior número de espécies exclusivas. Duas destas, Schizodon altoparanae e Salminus brasiliensis, são espécies nativas migradoras de médio a grande porte. Segundo Agostinho et al. (1999), a conseqüência de uma série de barragens nos principais tributários do alto rio Paraná tem sido a ausência de grandes migradores, demonstrando assim a importância da retenção de características originais do ambiente lótico, como aconteceu neste trecho.

Os gêneros Hypostomus e Loricaria também ocorreram somente em Cinzas. O caráter reofílico dos cascudos explica, pelo menos em parte, suas baixas freqüências em áreas represadas (BENNEMANN et al., 1995; BenEDito-CeCilio et al., 1997). A mais óbvia limitação do represamento é a restrição ou eliminação do acesso a longos trechos fluviais, e tem sido verificado que, para se reproduzir, a maioria das espécies que colonizam reservatórios na região neotropical procuram tributários laterais ou outras áreas lóticas (AgOstinHo et al., 1999), o que reduz os impactos negativos de um represamento sobre o comportamento reprodutivo (Agostinho et al., 1994). Isso foi demonstrado por SANTOS \& FoRMAGIO (2000) na bacia do rio Grande para um grande número de espécies de médio e grande porte. VAZZOLER et al. (1997) verificaram que espécies como Prochilodus lineatus, Salminus brasiliensis, Pimelodus maculatus e Leporinus elongatus, que também ocorrem no médio Paranapanema, desovam nos trechos superiores dos tributários do rio Paraná a montante do reservatório de Itaipu.

Os resultados do presente trabalho demonstraram a influência da barragem sobre a diversidade de peixes deste ambiente, pois quanto mais afastado de Porecatu (zona lacustre) em direção ao ambiente menos influenciado pelo barramento (ou seja, com características mais semelhantes ao ambiente original, que neste caso é o trecho de Cinzas), maior é a diversidade. Cinzas é o único ambiente lótico dentre os trechos amostrados, representando assim a zona fluvial do reservatório. O rio Cinzas pode ser considerado uma continuidade do reservatório de Capivara, uma vez que, logo acima da foz deste rio está a barragem de Canoas I. Um fator adicional que contribuiu com a riqueza no trecho Cinzas, portanto, foi a presença do tributário rio das Cinzas que demonstrou ter um papel importante para a manutenção da diversidade. Segundo Agostinho et al. (1999), a zona fluvial de um reservatório tem com frequêencia a maior diversidade de espécies por incluir as espécies típicas de ambientes lóticos, além das espécies que ocorrem nas zonas de transição e lacustre.

O mesmo fenômeno pode ser observado em Tibagi, por ser o maior tributário do reservatório, que na sequiência de Cinzas, é o segundo trecho com maior diversidade. Os resultados referentes à semelhança ictiofaunística vêm confirmar a grande similaridade entre os trechos Cinzas e Tibagi, que são influenciados pela presença de tributários de grande porte ligados ao 
reservatório e que estão mais distantes da barragem. A semelhança entre Porecatu e Cruzália se deve ao fato de constituírem-se em locais extremamente influenciados pela formação do reservatório e pela ausência de afluentes de grande porte. Entretanto, em Porecatu, apesar da maior proximidade com a barragem do que Cruzália, a diversidade é maior que este último por estar localizado na foz do rio Vermelho, que a despeito do pequeno porte ameniza os efeitos do represamento sobre este ambiente, inclusive com águas semi-lóticas e nãolênticas como seria neste trecho se não houvesse a presença deste rio.

Portanto, estes resultados demonstraram mais uma vez a importância de se considerar a presença de grandes tributários no local de construção de reservatórios e a preservação de remanescentes lóticos responsáveis pela diversidade original da bacia, para a manutenção de populações reofílicas e da diversidade da ictiofauna do reservatório.

Agradecimentos. À Universidade Estadual de Londrina pelo espaço físico; ao Programa de Pós-Graduação em Ciências Biológicas pelos recursos financeiros; à CAPES pela bolsa concedida; ao convênio Duke Energy/FAUEL pelo apoio financeiro. À Evanilde Benedito-Cecílio, Oswaldo T. Oyakawa, Francisco Langeani e Julio César Garavello pelas sugestões de grande valia para este trabalho.

\section{REFERÊNCIAS BIBLIOGRÁFICAS}

Agostinho, A. A. 1992. Manejo de recursos pesqueiros em reservatórios. In: Agostinho, A. A. \& Benedito-Cecilio, E. eds. Situação atual e perspectivas da ictiologia no Brasil. Maringá, EDUEM. p.106-121.

Agostinho, A. A. \& Gomes, L. C. 1997. Manejo e monitoramento de recursos pesqueiros: perspectivas para o Reservatório de Segredo. In: Agostinho, A. A. \& Gomes, L. C. eds. Reservatório de Segredo: bases ecológicas para o manejo. Maringá, EDUEM. p.319-364.

Agostinho, A. A.; Júlio JR., H. F. \& Borghetti, J. R. 1992. Considerações sobre os impactos dos represamentos na ictiofauna e medidas para sua atenuação - um estudo de caso: reservatório de Itaipu. Revista Unimar 14(suplemento):89107.

Agostinho, A. A.; Julio Jr., H. F. \& Petrere JR., M. 1994. Itaipu reservoir (Brazil): impacts of the impoudment on the fish fauna and fisheries. In: Cowx, I. G. ed. Rehabilitation of Freshwater Fisheries, Oxford, Fishing News Book. p.171184.

Agostinho, A. A.; Miranda, L. E.; Bini, L. M.; Gomes, L. C.; Thomaz, S. M. \& SUZuKI, H. I. 1999. Patterns of colonization in neotropical reservoirs, and prognoses on aging. In: TUNDISI, J. G. \& Straskraba, M. eds. Theoretical Reservoir Ecology and its Applications. São Carlos, International Institute os Ecology, Brazilian Academy of Sciences and Backhuys Publishers. p.227-265.

Araújo, F. G. \& Santos, L. N. 2001. Distribution of fish assemblages in Lajes reservoir, Rio de Janeiro, Brazil. Brazilian Journal of Biology 61(4):563-576.

Benedito-Cecilio, E. B.; Agostinho, A. A.; Júlio, H. F., JR. \& Pavanelli, C. S. 1997. Colonização ictiofaunística do reservatório de Itaipu e áreas adjacentes. Revista Brasileira de Zoologia 14(1):1-14.

Bennemann, S. T., Shibatta, O. A. \& Garavello, J. C. 2000.
Peixes do rio Tibagi: uma abordagem ecológica Londrina, UEL. 64p.

Bennemann, S. T., Silva-Souza, A. T. \& Rocha, G. R. A. 1995 Composicion ictiofaunistica en cinco localidades de la cuenca del Rio Tibagi, PR - Brasil. Interciencia 20(1):7-13.

Britski, H. A. 1972. Peixes de água doce do estado de São Paulo. In: Comissão Internacional da Bacia Paraná - Paraguai Poluição e piscicultura. São Paulo, Faculdade de Saúde Pública da USP e Instituto de Pesca. p.79-108.

Carvalho, E.; Silva, V. F. B.; Fujihara, C. Y.; Henry, R. \& Foresti, F. 1998. Diversity of fish species in the Paranapanema river - Jurumirim reservoir transition region (São Paulo State, Brasil). Italian Journal of Zoology 65:325-330.

CESP. 1993. Informações gerais. São Paulo, CESP (Companhia Energética de São Paulo). 73p.

Cox-Fernandes, C.; Podos, J. \& Lundberg, J. G. 2004. Amazonian ecology: tributaries enhance the diversity of electric fishes. Science 305(5692):1960-1962.

DAjoz, R. 1978. Ecologia Geral. 3 ed. São Paulo, Vozes, EDUSP. 474p.

Dias, J. H. P. \& Garavello, J. C. 1998. Ecological studies on the fish community of Salto Grande Reservoir, Paranapanema River Basin, São Paulo, Brazil. Verhandlungen International Vereinigung Limnology 26:2228-2231.

Ferreira, E. J. G. 1993. Composição, distribuição e aspectos ecológicos da ictiofauna de um trecho do rio Trombetas, na área de influência da futura UHE Cachoeira Porteira, Estado do Pará, Brasil. Acta Amazonica 23(Supl.1/4):1-88.

Hammer, Ø.; Harper, D. A. T. \& Ryan, P. D. 2003. Past Palaentological Statistics, ver. 1.12. Disponível em: <http:/ /folk.uio.no/ohammer/past>. Acesso em: 31.07.2003.

Lowe-Mconnell, R. H. 1999. Estudos Ecológicos de Comunidades de Peixes tropicais. São Paulo, EDUSP. 534p.

MAACK, R. 1981. Geografia Física do Estado do Paraná. 2 ed. Rio de Janeiro, José Olympio. 450p.

Magurran, A. 1988. Ecological Diversity and its Measurement. London, Croom Helm. 179p.

Orsi, M. L.; Shibatta, O. A. \& Silva-Souza, A. T. 2002. Caracterização biológica de populações de peixes do rio Tibagi, localidade de Sertanópolis. In: Medri, M. E.; Shibatta, O. A.; Bianchint, E. \& Pimenta, J. A. eds. A Bacia do Rio Tibagi. Londrina, Edição dos Editores. p.425-432.

Sampaio, T. 1944. Relatório sobre os estudos efetuados nos rios Itapetininga e Paranapanema. Revista do Instituto Geográfico e Geológico 2(3):30-81.

SANTOS, G. M. 1995. Impactos da hidrelétrica Samuel sobre as comunidades de peixes do Rio Jamari (Rondônia, Brasil). Acta Amazonica 25:247-280.

Santos, G. B. \& Formagio, P. S. 2000. Estrutura da ictiofauna dos reservatórios do rio Grande, com ênfase no estabelecimento de peixes piscívoros exóticos. Informe Agropecuário 21(203):98-106.

SCHAEFFER, S. A. 1998. Conflict an Resolution: Impact of new taxa on phylogenetic studies of the neotropical cascudinhos (Siluroidei: Loricariidae). In: Malabarba, L. R.; Reis, R. E.; Vari, R. P.; Lucena, Z. M. S. \& Lucena, C. A. S. Phylogeny and classification of Neotropical fishes. Porto Alegre, EDIPUCRS, p.375-400.

SNeAth, P. H. A. \& SoKal, R. R. 1973. Numerical taxonomy: the principles and pratice of numerical classification. San Francisco, W.H. Freeman and Company. 573p.

Vazzoler, A. E. A. M.; Suzuki, H. I.; Marques, E. E. \& Lizama, M. A. 1997. Primeira maturação gonadal, períodos e áreas de reprodução. In: VAzzoler, A. E. A. M; Agostinho, A. A. \& HoHn, N. S. eds. A planície de inundação da alto Paraná: aspectos físicos, biológicos e sócio-econômicos. Maringá, UEM. p. 249-265.

ZocchI, P. 2002. Paranapanema: da nascente à foz. São Paulo, Audichromo, 132p. 